\title{
New Methodology for Mixed Simulation of Active Antenna
}

\author{
G. ZAKKA EL NASHEF, F. TORRES, S. MONS, T. REVEYRAND T. MONÉDIÈRE, E. NGOYA, M. \\ THEVENOT, R. QUERE
}

XLIM - C2S2/OSA UMR CNRS nº6172 Limoges, France

\begin{abstract}
This paper presents a mixed simulation approach for an active antenna operating at $8.2 \mathrm{GHz}$. This approach is composed of three main parts which they will be detailed and described with some experimental validation as well. The first part of this approach is an electromagnetic (EM) macromodel for antennas array. The second part is a bilateral behavioral model for power amplifiers (PA). Finally, a mixed simulation approach was elaborated and implemented into a circuit simulator using both developed models. Theoretical and experimental results show the reliability of EM, PA models and the mixed simulation approach as well. The originality of this work is the elimination of isolator between PA and antennas while maintaining a correct radiation pattern despite high antenna impedance mismatches.
\end{abstract}

Keywords: Active Electronically Scanned Array (AESA); behavioral modeling; impedance mismatch; nonlinear scattering parameters; power amplifiers.

\section{INTRODUCTION}

Today's requirement in AESA applications, in terms of size, performance, weight and cost, are becoming serious constraints where low-profile systems are required [1], [2]. In some applications and in the framework of our studies, single transmission (TX) chains are desirable. In such chains, the last three traditional components are the power amplifier (PA), isolator and antenna. Isolator's important role is to minimize PA load variations and preserve linearity. Unfortunately, isolators are bulky devices and do not address the low cost and size reduction requirements. If the isolator is to be eliminated, interaction between PA and antenna has to be accurately predicted and controlled, with special focus on the mismatching due to the mutual coupling between antennas. This mismatching clearly affects PA performances in terms of gain $(\mathrm{AM} / \mathrm{AM})$ and phase $(\mathrm{AM} / \mathrm{PM})$, thus modifying the necessary weights applied to the array and degrading its efficiency and radiation performance. Moreover, these consequences can lead to instability or breakdown of PA output stage due to large VSWR (Voltage Standing Wave Ratio), making the mismatching effect a major challenge and that must be taken into account. Consequently, a mixed simulation approach of EM macromodel and nonlinear circuit model has to be developed to ensure that the overall performance will meet requirements in given worst-case conditions.

This paper describes each part of this mixed simulation approach. Various theoretical and experimental results show the reliability of EM and PA models and of the mixed simulation approach as well. The originality of this work is the elimination of isolator between PA and antennas while maintaining a correct radiation pattern despite high antenna impedance mismatches, i.e. VSWR.

\section{EM MACROMODEL}

\section{A. Description}

One of the most critical factors in antennas array is the mutual coupling between inter-elements where several approaches have been conducted to take into account this phenomenon [3]. Our method, i.e. the EM macromodel, is based on the calculation of active element patterns [4], which takes into account the mutual coupling between elements. The calculation of these active patterns is obtained from CST MS along with the current and voltage matrix for the whole array.

To be more precise, the EM macromodel is based on the electrical schematic shown in Fig. 1. It is composed of three parts, the first one is the generator characterized by its intrinsic impedance $\left(Z_{\mathrm{Li}}\right)$, the second part is the antenna characterized by its proper impedance $\left(Z_{\mathrm{ei}}\right)$ and the last part is the induced currents $\left(\mathrm{I}_{0^{\prime}} \mathrm{i}\right)$, which characterize the mutual coupling, from other elements in the array.

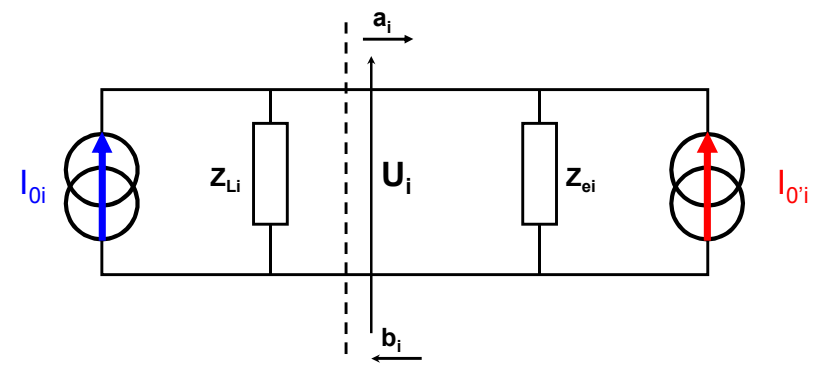

Figure 1. Equivalent electrical schematic.

The main challenge in this section is to establish an EM macromodel which is able to take into account the mutual coupling, calculate the active impedance $\left(Z_{\text {active }} \neq 50 \Omega\right)$ while taking into account the optimal matching $Z_{\text {active } i}=Z_{\mathrm{Li}^{*}}$ and calculates the feeding weights (" $a_{i}$ " in terms of magnitude and phase) in order to achieve the desired radiation pattern [5], [6]. When these conditions are fulfilled correctly, the coupled power (" $b_{i}$ "), as shown in Fig. 1, is almost equal to zero and will not disturb the array or PA functioning. Thus, 
the isolator becomes useless in the TX chain. The experimental validation described in section III will prove the reliability of this concept. Moreover, the whole incident power is transmitted to the antennas and radiation efficiency is maximized.

The calculation procedure for the EM macromodel is composed of three steps and implemented in matlab. The first step consists of calculating the reflection coefficients $\left(\alpha_{\mathrm{ij}}\right)$ and the antennas impedances $\left(\mathrm{Z}_{\mathrm{ei}}\right)$ for the antennas array, by using the data obtained from CST full wave simulation (voltage and current matrix). The second step uses the frequency and the pointing angle as inputs in order to define a desired array radiation pattern which was obtained from an optimization tool, called SARA, developed in our laboratory [7]. Finally, the active impedances $\left(Z_{\text {active }}\right)$ and their corresponding weights are calculated to fit the radiation pattern, defined by SARA. All the mathematical formulation and the EM macromodel development can be found in [8]. The next paragraph will show some theoretical and experimental validation in order to validate the EM macromodel.

\section{B. Theoretical and experimental results}

In order to test the robustness and the accuracy of the EM macromodel, some theoretical validations were achieved. We have selected three different examples of patch arrays; the first one is a linear array of 16 elements (linear polarization) working at $2.45 \mathrm{GHz}$ with inter-element spacing equal to $0.4 \lambda_{0}$. The second example is a linear array of 8 elements (circular polarization) working at $5.5 \mathrm{GHz}$ with inter-element spacing equal to $0.65 \lambda_{0}$ and the last example is a planar array with $8 \times 8$ elements working at $8.2 \mathrm{GHz}$ with inter-element spacing equal to $0.4 \lambda_{0}$. Fig. 2 , Fig. 3 and Fig. 4 present the comparison between the simulation and the developed EM macromodel.

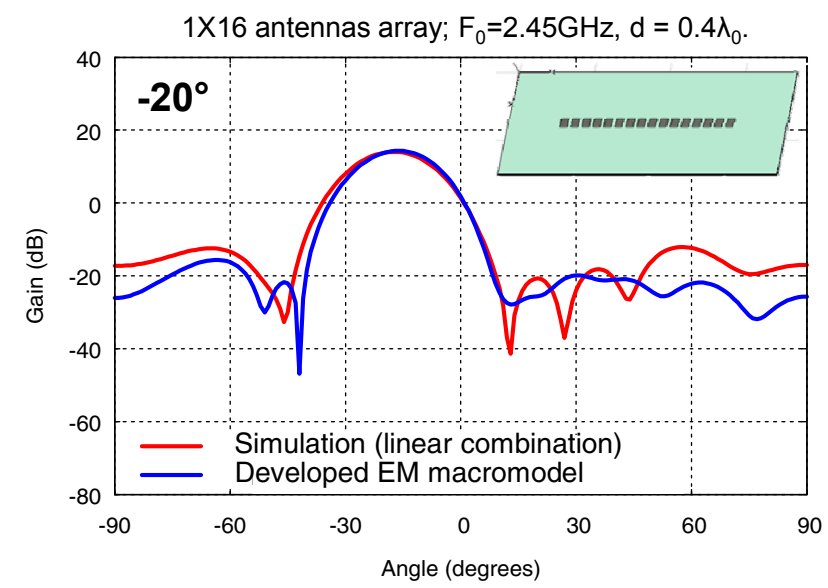

Figure 2. Comparison between the simulation and the macromodel results for a 1 X16 linear array working @ $2.45 \mathrm{GHz}$ and a pointing angle of $-20^{\circ}$.

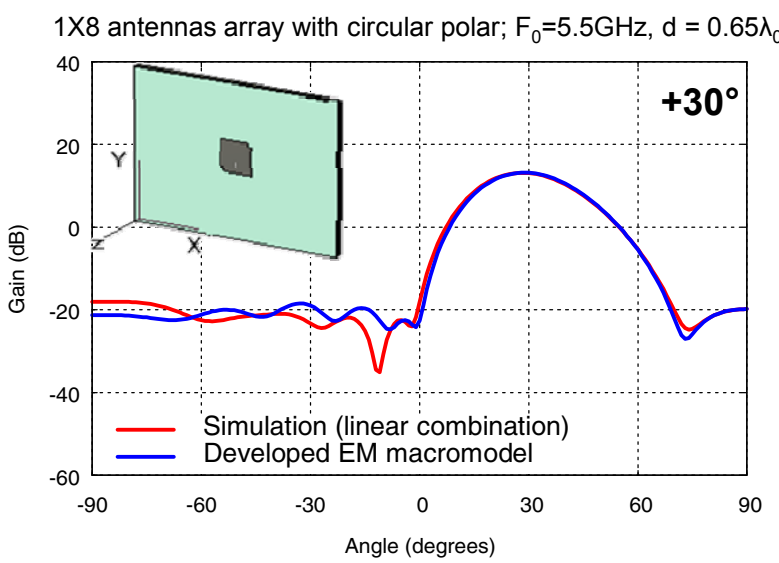

Figure 3. Comparison between the simulation and the macromodel results for a 1X8 linear array with circular polar, working@2.45 GHz and a pointing angle of $+30^{\circ}$.

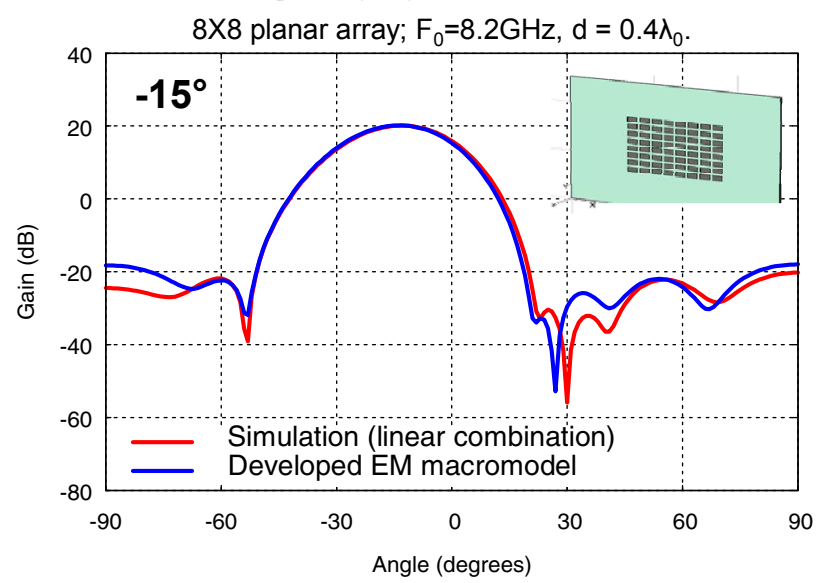

Figure 4. Comparison between the simulation and the macromodel results for an 8X8 planar array working @ $8.2 \mathrm{GHz}$ and a pointing angle of $-15^{\circ}$.

The theoretical results above show great performance concerning the macromodel efficiency. The EM macromodel is validated for any pointing angle, any working frequency, any array size $(\mathrm{NxM})$ and any type of polarization (linear, circular). Moreover, these results also show that the EM macromodel can be used as a synthesis tool.

An experimental validation is needed to assess the theoretical aspect of this EM macro-model; therefore we decided to use attenuators and phase shifters in order to control the weights, which are calculated by the EM macromodel, in terms of magnitude and phase on each antenna access of the array. The EM demonstrator is composed of power divider, attenuators, phase shifters and $1 \mathrm{X} 8$ antennas array (Fig. 5). Two pointing angles $\left(-20^{\circ}\right.$ and $+30^{\circ}$ ) were measured. Fig. 6 and Fig. 7 present the comparison between the corresponding rigorous simulations, i.e. linear combination solver on CST MS and the measurement for the different pointing angles. The working frequency is $8.2 \mathrm{GHz}$. 


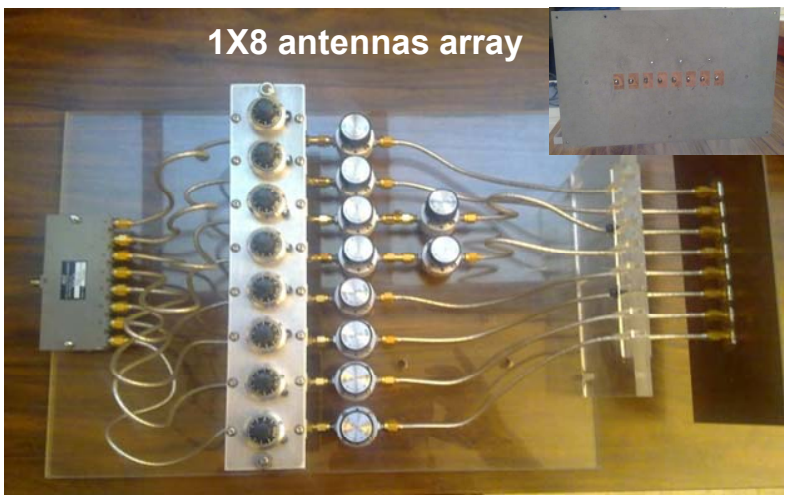

Figure 5. EM demonstrator



Figure 6. Radiation pattern comparison $\left(-20^{\circ}\right)$. EM macromodel simulation (solid line). Measurement (dashed line). $\mathrm{F}_{0}=8.2 \mathrm{GHz}$.



Figure 7. Radiation pattern comparison $\left(+30^{\circ}\right)$. EM macromodel simulation (solid line). Measurement (dashed line). $\mathrm{F}_{0}=8.2 \mathrm{GHz}$.

Fig. 6 and Fig. 7 show a very good agreement between the simulation and the measurement results especially in terms of main lobes. However, we can notice some slight differences on the side lobe levels due to some uncertainties in the anechoic chamber. We can conclude, in this section, that the obtained results prove the efficiency and strength of the EM macromodel. Moreover, this EM macromodel can also be integrated in a circuit simulator, e.g. ADS from Agilent, in order to study the global interactions between antennas and PA.

\section{PA BEHAVIORAL MODEL}

\section{A. Description}

As for the antennas array and their critical effect, i.e. the mutual coupling, another crucial effect, i.e. the mismatching effect, appears in PA behavior and therefore must be taken into account. Moreover, PA undergoes many disturbances in active antennas applications, such as high frequency (shortterm) and low frequency (long-term) memory effects, thermal effects and mismatching effect. However, our main focus requires studying correctly the interaction between antennas and PA in order to quantify their impact on TX chains where the mismatching effect can directly impact the gain and the phase of each antenna access leading to an accurate radiation pattern. The short relative distance between the array's elements may result in a high level of mutual coupling between antennas, leading to large output loading mismatches (up to VSWR $=4$ and more). Therefore, an accurate PA model is needed to predict its' electrical performance and study the mismatching influence on PA performance.

Several models have been proposed to encounter this problem in PA [9], [10] thanks to a formalism called nonlinear scattering functions, introduced in [11]. The nonlinear scattering functions consist in defining a nonlinear relation for the $[\mathrm{S}]$ parameters:

$$
\widetilde{b}=\left[S_{i j}\left(\left|a_{1}\right|\right)\right] \cdot \widetilde{a}
$$

Where $\quad \tilde{a}=\left[a_{1}, a_{2}, a_{2}^{*}\right]^{T}$ and $\tilde{b}=\left[b_{1}, b_{2}\right]^{T}$ are respectively the incident and reflected power waves, and $S_{i j}\left(\left|a_{1}\right|\right), i=1,2 ; j=1,2$ are the nonlinear scattering functions. However, these models are only a linear approximation in regards to the output incident wave " $\mathrm{a}_{2}$ ". As a consequence, the accuracy of the model can be very limited in large output mismatch conditions (VSWR > 3), where " $a_{2}$ " becomes large. In [8], [12], we developed an extended behavioral model of (1) which allows to better reproduce PA mismatch behavior for relatively large VSWR, typically up to 4 . Such accuracy is necessary to correctly model the PA feeding the radiating elements in AESA applications.

The developed behavioral model in [8] can be extracted from simple continuous wave $(\mathrm{CW})$ measurements of $\mathrm{PA}$, as described in [13]. The extraction of the nonlinear parameters, the development and the implementation in ADS for this model can be found in [8], [12]. Briefly, the measurement setup is a conventional load pull bench, where the identification of nonlinear scattering functions consists of measuring the incident and reflected power waves driven by a CW signal at the operating frequency, e.g. $8.2 \mathrm{GHz}$, of the PA in a load-pull environment. The measured data will acquire complete and accurate information of PA electrical behavior which enable us to define a nonlinear behavioral model, as explained in [8]. The implementation of the model is established into ADS by using a "black Box" circuit model. The annotation "black box" refers to the fact that no knowledge is used nor required concerning the internal circuitry of the device under test (PA). The model has been 
implemented thanks to an FDD (Frequency-domain Defined Device) nonlinear block.

\section{B. Experimental results}

To validate the model, we have considered a commercial 8-14 GHz PA from NEXTEC RF (NEXTEC-RF NB00422). It exhibits a $17 \mathrm{~dB}$ small signal gain with a $27 \mathrm{dBm}$ output power at $1 \mathrm{~dB}$ gain compression and an associated power added efficiency of $17 \%$. The extraction of the nonlinear $\mathrm{S}$ parameter (after the PA measurement) is done using a developed code in Scilab. Then, the PA model is implemented in ADS along with the measurement in order to compare them, as described in [8].

Fig. 8 shows the comparison between the second order model and the measurement in terms of gain (AMAM) and phase (AMPM) for different VSWR. This test under large load mismatches will attest the second order model accuracy and the prediction ability of PA model.

A AMAM Measurement @ Z1 (VSWR=2)

- AMAM Measurement @ Z2 (VSWR=3)

- AMAM Measurement @ Z3 (VSWR=4)

- AMPM Measurement @ Z1 (VSWR=2)

- AMPM Measurement @ Z2 (VSWR=3)

- AMPM Measurement @ Z3 (VSWR=4)

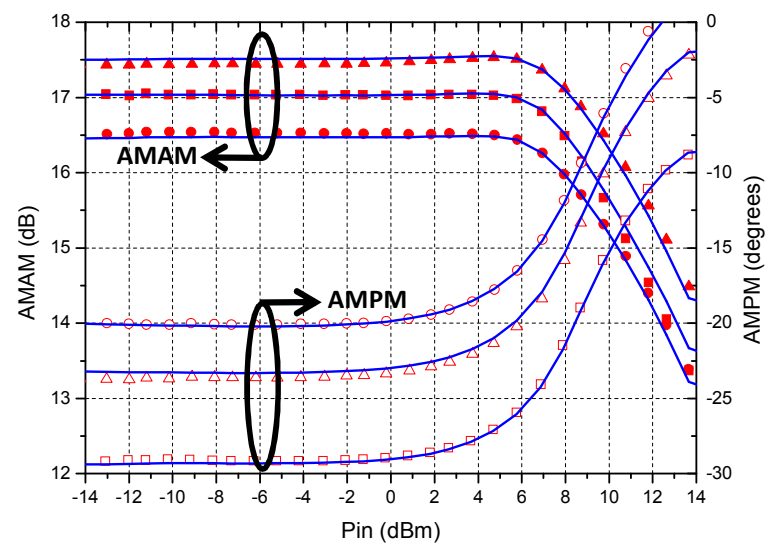

Figure 8. AMAM and AMPM vs. input power for different VSWR $(Z 1=$ $36.8-\mathrm{j} \cdot 27.1, \mathrm{Z} 2=35.2-\mathrm{j} \cdot 5.8, \mathrm{Z3}=14.8+\mathrm{j} \cdot 0.4)$. Measurement (symbols) and model (solid line). $\mathrm{F}_{0}=8.2 \mathrm{GHz}$.

We can notice significant changes of PA performance over the three impedances of various VSWR. These results indicate the importance to take into account the mismatching effect. Otherwise, Fig. 8 show a very good agreement between PA model and PA measurements, showing the prediction ability of PA model to take into account moderate VSWR at the price of a small degradation of performances.

\section{MIXED SIMULATION APPROACH}

\section{A. Description}

Once both models (EM model and PA model) are developed, a mixed simulation approach is elaborated consisting of the combination of the two developed model in a circuit simulator, e.g. ADS from Agilent. The concept is to use the calculated active impedances, obtained from the EM macromodel, for each antenna access in the array at different pointing angles, e.g. $0^{\circ},-20^{\circ},+30^{\circ}$, as load impedances for the PA. In that case, PA overall performance is studied by simulation, i.e. the implemented model in ADS, and the measurement, i.e. the load-pull bench described in [13], for some particular impedances which characterize the antennas. Fig. 9 shows the comparison between PA model (implemented into ADS) and measurements in terms of gain (AMAM) and phase (AMPM) for three different antennas impedances calculated by the EM macro-model.

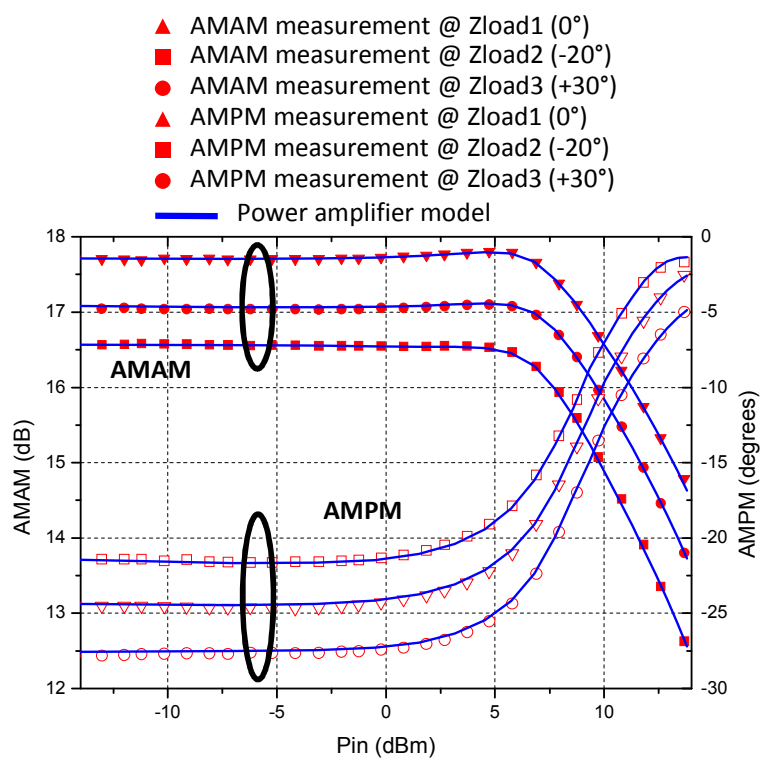

Figure 9. AMAM and AMPM vs. input power for calculated EM model impedances $(\mathrm{Z}$ load $1=46.1+\mathrm{j} \cdot 9.7, \mathrm{Z}$ load $2=42.3+\mathrm{j} \cdot 21.5, \mathrm{Z}$ load $3=$ $25.5+\mathrm{j} \cdot 18.5$ ). Measurement (symbols) and model (solid line). $\mathrm{F}_{0}=8.2 \mathrm{GHz}$.

Fig. 9 shows a perfect agreement, and this again demonstrates the efficiency and accuracy of PA model, even in the presence of output loading impedances characterizing the antennas.

The obtained results from the mixed simulation, i.e. the combination of both models in ADS, enable us to acquire important knowledge in terms of magnitude (AMAM) and phase (AMPM) variations (difference between the PA output and input). The acquired data will allow us to adjust PA input in order to correct the feeding weights of the array to finally obtain the desired radiation pattern, and enables robust, accurate and useful simulation of distortion at circuit level. The next paragraph presents an active antenna demonstrator showing the feasibility of realizing a TX chain without an isolator while maintaining an optimum radiation pattern. 


\section{B. Experimental results}

Besides developing and validating two robust and effective models which take into account the mutual coupling and the mismatching. We were able, as well to optimize the radiation pattern of the array in directivity, gain and side lobes level and to obtain a reflected power wave $\left(b_{i}\right)$ almost equal to zero, which will not disturb the array neither PA functioning. However, one small hypothesis remains: the possibility to eliminate isolators between PA and antennas. Therefore an active antenna demonstrator was realized to demonstrate the effectiveness of the proposed mixed simulation approach and prove the feasibility of this hypothesis.

Fig. 10 depicts the active demonstrator where we used a power divider connected to attenuators and phase shifters. PA are now directly inserted between the phase shifters and array's antennas without the use of isolators. A set of measured results with $0^{\circ},-20^{\circ}$ and $+30^{\circ}$ are presented in Fig. 11, Fig. 12 and Fig. 13. They compare the measured radiation patterns with PA (active antenna demonstrator), the measured radiation patterns without PA (EM macro-model validation), and simulated radiation patterns without PA (CST) for $0^{\circ},-20^{\circ}$ and $+30^{\circ}$ pointing angles.



Figure 10. Active antenna demonstrator

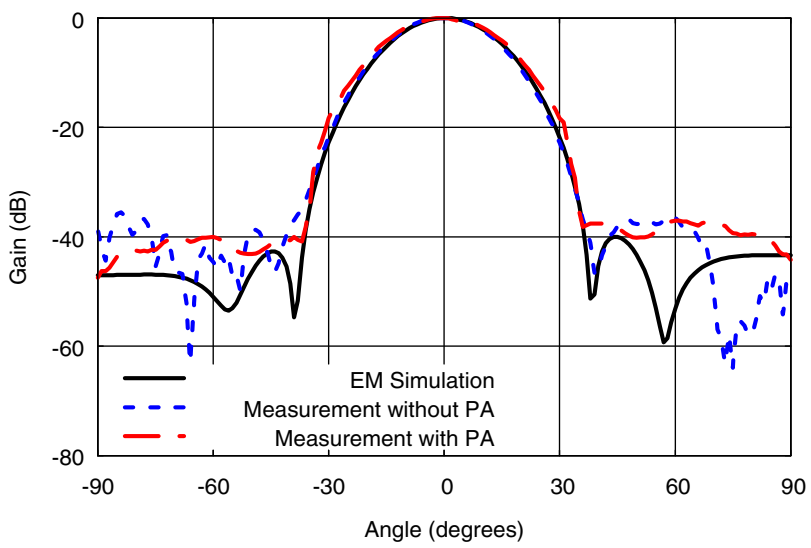

Figure 11. Radiation pattern comparison $\left(0^{\circ}\right)$. EM macromodel simulation (solid line). Measurement without PA (small dashed line). Measurement with PA (big dashed line). $\mathrm{F}_{0}=8.2 \mathrm{GHz}$

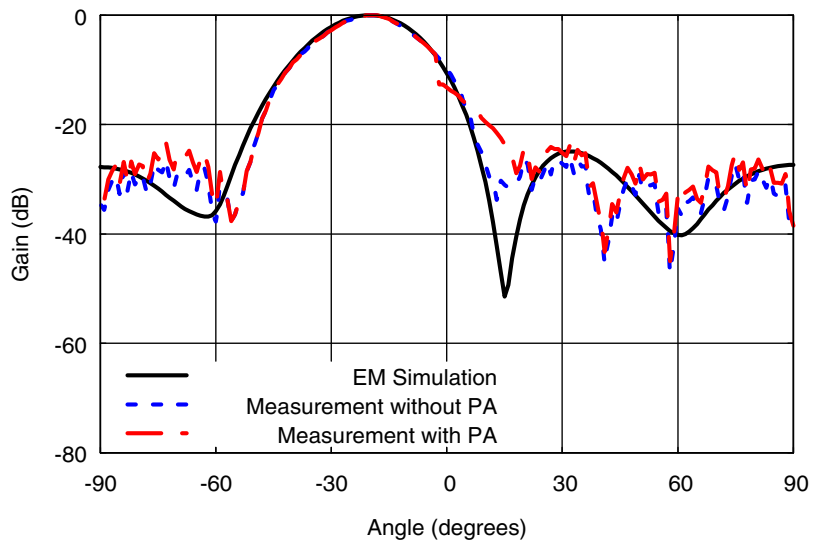

Figure 12. Radiation pattern comparison $\left(-20^{\circ}\right)$. EM macromodel simulation (solid line). Measurement without PA (small dashed line). Measurement with PA (big dashed line). $\mathrm{F}_{0}=8.2 \mathrm{GHz}$.

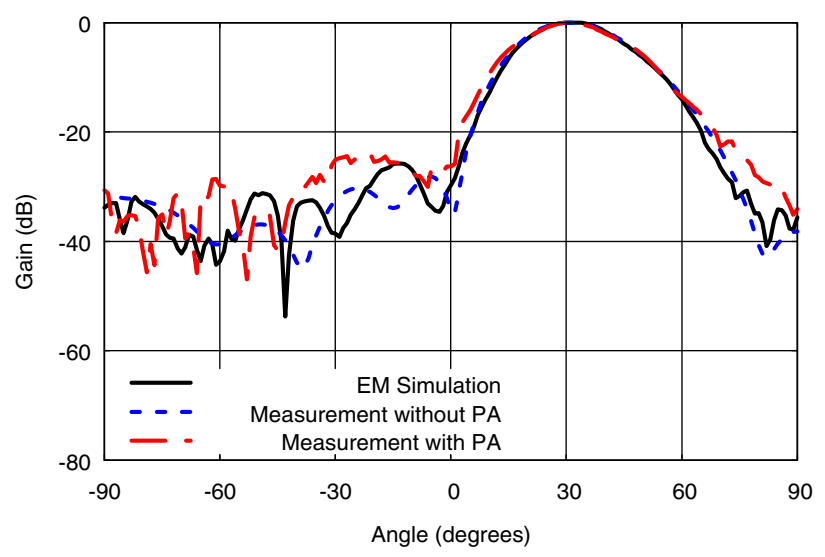

Figure 13. Radiation pattern comparison $\left(+30^{\circ}\right)$. EM macromodel simulation (solid line). Measurement without PA (small dashed line). Measurement with PA (big dashed line). $\mathrm{F}_{0}=8.2 \mathrm{GHz}$.

The results above show a very good agreement between the measured radiation patterns and the simulated radiation patterns despite some slight differences on side lobe levels which are likely caused by the inaccuracies during the measuring procedure. However, our hypothesis (no use of isolators between PA and antennas) has been fulfilled.

\section{CONCLUSION}

This paper presented a robust approach, called "mixed simulation", which enables us to study the direct connection between PA and antennas. It consists of developing two models, one called the EM macromodel which takes into account the mutual coupling for an antennas array. The other called the extended PA behavioral model which takes into account the mismatching effects related to PA. Both models were validated experimentally. Finally, the mixed simulation approach was elaborated from the combination of both models into a circuit simulator (ADS) and was validated using an active antenna demonstrator. The originality of this work was the elimination of an isolator from a TX chain while providing a robust solution to new designs verification capabilities for active antennas applications. 


\section{REFERENCES}

[1] Hommel, H.; Feldle, H.P, “ Current status of airborne active phased array (AESA)", radar system and future trends, 34th European Microwave Conf., Amsterdam, 2004, 1517-1520.

[2] L. Sankey, Z. Popovic, "Adaptive tuning for handheld transmitters", IEEE MTT-S Int. Microwave Symp. Digest, June 2009.

[3] C. A. Balanis, "Antenna Theory Analysis and Design", Wiley Interscience, 3rd edition, 2005.

[4] Pozar, D.M, "The active element pattern", IEEE Trans. Antennas Propag., 42 (8) (1994), 1176-1178.

[5] D.F. Kelly and W.L. Stutzman, "Array antenna pattern modeling methods that include mutual coupling effects", IEEE Transactions on Antennas and Propagation, Vol.41, n ${ }^{\circ} 12$, pp.1625-1632, 1993.

[6] D.M. Pozar, "A relation between the active input impedance and the active element pattern of a phased array", IEEE Transactions on Antennas and Propagation. Vol.51, n 9, pp.2486-2489, 2003.

[7] M. Mouhamadou, N. Fadlallah, P. Vaudon, M. Rammal, "Adaptive Beamforming Synthesis for Linear Antenna Array Controlling only by Phase with Interference Canceller", LAPC 2006, Loughborough Antennas \& Propagation Conference, Loughborough University, UK, PP. 165-168, 11-12 April 2006.

[8] G. Zakka El Nashef, F. Torres, S. Mons, T. Reveyrand, E. Ngoya, T. Monedière, M. Thévenot, R. Quéré, "An accurate modeling technique for antennas and nonlinear RF power amplifier mixed simulation", International Journal of Microwave and Wireless Technologies, volume 3, issue 6, December 2011, pp. 647-655.

[9] D.E. Root, J. Verspecht, D.sharrit, J. Wood, A. Cognata, "BroadBand Poly-Harmonic Distorsion (PHD) Behavioral Models from Fast Automated Simulations and Large-Signal Vectorial Network Measurement", IEEE Transactions on Microwave Theory and Techniques Vol. 53, n¹1,pp. 3656-3664, Nov. 2005.

[10] A. Soury, E. N'Goya, J. Rousset, "Behavioral Modeling of RF and Microwave Circuit Blocks for Hierarchical Simulation of Modern Transcievers", IEEE MTT-S Int. Microwave Symp. Digest, June 2005 pp. 975-978.

[11] J.Verspecht, "Scattering Functions for Nonlinear Behavioral Modeling in the frequency domain", Fundamentals of Nonlinear Behavioral Modeling, Foundations and Applications workshop, IEEE MTT-S, International Microwave Symposium, June 2003.

[12] G. Zakka El Nashef, F. Torrès, S. Mons, T. Reveyrand, T. Monédière, E.Ngoya, R. Quéré, "Second Order Extension of Power Amplifiers Behavioral Models for Accuracy Improvements", European Microwave Week, Paris, France, 27-29 Sep, 2010.

[13] T. Reveyrand, T. Gasseling, D. Barataud, S. Mons, J-M. Nébus, “A Smart Load-Pull Method to Safely Reach Optimal Matching Impedances of Power Transistors", IEEE MTT-S Digest, Honolulu, Hawaii, June 2007, pp.1489-1492. 